\title{
Naldemedine in Japanese patients with opioid- induced constipation and chronic noncancer pain: open-label Phase III studies
}

This article was published in the following Dove Press journal: Journal of Pain Research

\author{
Yoji Saito' \\ Takaaki Yokota ${ }^{2}$ \\ Masatsugu $\mathrm{Arai}^{3}$ \\ Yukio Tada ${ }^{4}$ \\ Masahiko Sumitani ${ }^{5}$ \\ 'Department of Anesthesiology, \\ Shimane University Hospital, \\ Shimane, Japan; ${ }^{2}$ Project Management \\ Department, Shionogi \& Co., Ltd, \\ Osaka, Japan; ${ }^{3}$ Business Development \\ Department, Shionogi \& Co., Ltd, \\ Osaka, Japan; ${ }^{4}$ Biostatistics Center, \\ Shionogi \& Co., Ltd, Osaka, Japan; \\ ${ }^{5}$ Department of Pain and Palliative \\ Medicine, The University of Tokyo \\ Hospital, Tokyo, Japan
}

Introduction: Naldemedine is a peripherally-acting $\mu$-opioid-receptor antagonist, approved in Japan for opioid-induced constipation (OIC). In two open-label, single-arm, Phase III studies, we evaluated the safety and efficacy of naldemedine in Japanese patients with OIC receiving regular-use opioids (COMPOSE-6) or prolonged-release oxycodone (COMPOSE-7) for chronic noncancer pain. Methods: Eligible Japanese adults with OIC and chronic noncancer pain received once-daily oral naldemedine $0.2 \mathrm{mg}$ for 48 weeks, irrespective of food intake. Primary end points included measures of treatment-emergent adverse events (TEAEs), pain intensity, and opioid withdrawal. Secondary efficacy end points were evaluated at treatment week 2. Patient Assessment of Constipation Symptoms (PAC-SYM) and Quality of Life (PAC-QOL) scores were evaluated in both 48-week studies. Results: Of patients enrolled in COMPOSE-6 $(\mathrm{N}=43)$ and COMPOSE-7 $(\mathrm{N}=10)$, TEAEs were reported in $88 \%$ (95\% CI 74.9-96.1) and 90\% (95\% CI 55.5-99.7), respectively. The most frequently reported TEAEs, nasopharyngitis and diarrhea, were mostly mild or moderate in severity. Assessments of pain intensity and opioid withdrawal remained stable over the 48-week treatment periods of both studies. The proportion of spontaneous bowel-movement responders at week 2 in COMPOSE-6 was 81.0\% (95\% CI 65.9-91.4) and 90.0\% (95\% CI 55.5-99.7) in COMPOSE-7. Significant and sustained improvements in PAC-SYM and PAC-QOL scores were also observed in both studies (all $P<0.05$ ).

Conclusion: Side effects that occurred with naldemedine were mostly mild or moderate in severity, and the data suggested that naldemedine can improve bowel function and QOL in Japanese patients with OIC receiving regular-use opioids or prolonged-release oxycodone for chronic noncancer pain.

Keywords: opioids, peripherally-acting $\mu$-opioid-receptor antagonists, patient assessment of constipation QOL, PAC-QOL, patient assessment of constipation symptoms, PAC-SYM, clinical studies, bowel function

\section{Plain language summary}

Opioids can help decrease the feeling of pain, but they can also induce constipation as a side effect. This side effect is termed opioid-induced constipation (OIC). Naldemedine is a drug that was recently approved to treat OIC. We administered naldemedine to Japanese patients with OIC who were receiving opioids for chronic noncancer pain. Naldemedine treated OIC in these patients by improving bowel function. Moreover, naldemedine did not raise any major safety concerns. The findings from our studies support the use of naldemedine to treat patients with OIC and chronic noncancer pain.

Correspondence: Takaaki Yokota Project Management Department, Shionogi \& Co., Ltd, Hankyu Terminal Bldg, I2th floor, I-I-4 Shibata, Kita-ku, Osaka 530-0012, Japan

Tel $+8 \mathrm{I} 664855089$

$\mathrm{Fax}+81663755780$

Email takaaki.yokota@shionogi.co.jp

\section{Introduction}

Opioid analgesics are prescribed for the management of chronic pain, which has an estimated prevalence of $39 \%$ in the general population of Japan. ${ }^{1,2}$ The use of opioids 
is commonly associated with debilitating side effects, such as opioid-induced constipation (OIC). ${ }^{3,4}$ Approximately $40 \%-60 \%$ of patients receiving opioids for chronic noncancer pain develop OIC., 5 The burden of OIC can adversely affect adherence to a prescribed opioid regimen, which can consequently impede adequate management of pain. ${ }^{7,8}$ Correspondingly, patients with OIC report a poor quality of life (QOL).,8

The binding of opioids to $\mu$-opioid receptors in the gastrointestinal tract alters enteric neural output, which in turn induces physiological changes, such as impaired gut motility and decreased fluid secretion, which contribute to the manifestation of OIC. ${ }^{9}$ Despite prevalent use, conventional laxatives are typically ineffective, because they do not target the underlying mechanism of OIC. ${ }^{9,10}$ Additionally, laxatives can further diminish QOL in patients with OIC. ${ }^{11}$ A combination regimen of prolonged-release (PR) oxycodone hydrochloride and the $\mu$-opioid-receptor antagonist naloxone has been found to decrease the incidence of OIC significantly in patients with chronic noncancer pain. ${ }^{4}$ However, when taken in high doses, naloxone can cross the blood-brain barrier, ${ }^{12}$ and there have been multiple case reports on negation of the analgesic benefits of PR oxycodone and precipitation of opioid-withdrawal syndrome in patients with compromised liver function. ${ }^{13-15}$

Naldemedine, a peripherally-acting $\mu$-opioid-receptor antagonist (PAMORA), is approved for the treatment of OIC in Japan ${ }^{16}$ and in adults with chronic noncancer pain in the US. ${ }^{16}$ Unlike that of naloxone, the structure of naldemedine limits the drug's ability to cross the blood-brain barrier and impact activity in the central nervous system. ${ }^{17}$ Accordingly, multiple global Phase III clinical studies have demonstrated the safety and efficacy of naldemedine in treating OIC without hindering the analgesic benefits of opioid therapy or inducing signs or symptoms of opioid withdrawal in patients with chronic noncancer pain or cancer. ${ }^{18,19}$

The long-term safety of coadministering naldemedine and PR oxycodone or other opioids in Japanese patients with chronic noncancer pain has yet to be determined. In Japan, PR oxycodone is indicated for the management of cancer pain, but has yet to be approved for chronic noncancer pain. ${ }^{20,21}$ Here, we report results from two open-label Phase III clinical studies that evaluated the long-term safety and efficacy of naldemedine in Japanese patients with OIC receiving regularuse opioids (COMPOSE-6) or PR oxycodone (COMPOSE-7) for chronic noncancer pain.

\section{Methods}

The COMPOSE-6 and COMPOSE-7 studies were singlearm, open-label, Phase III clinical studies. COMPOSE-6 was conducted across 21 sites, and COMPOSE-7 was conducted across nine sites, all in Japan. Both studies were approved by the respective institutional review boards (IRBs). IRBs for COMPOSE-6 and COMPOSE-7 were Nihon University Hospital Central IRB, Kondo Memorial Medical Foundation Tomisaka Clinic IRB, Ishikawa Prefectural Central Hospital IRB, Shiga University of Medical Science Hospital IRB, Nishinomiya Municipal Central Hospital IRB, Yasuda Hospital Review Board, Faculty of Medicine, University of Miyazaki Hospital Review Board, and the University of Tokyo Hospital Review Board. IRBs for COMPOSE-6 only were Yasuhiko Munakata Clinic IRB, Yamagata University Hospital IRB, Tokyo Metropolitan Health and Medical Corporation Ebara Hospital IRB, Chiba University Hospital IRB, Fujisawa City Hospital IRB, Niigata City General Hospital IRB, Toyama University Hospital IRB, the Japanese Association for the Promotion of State of the Art in Medicine IRB, Nissay Hospital IRB, Asakura Hospital Review Board, Saga University Hospital IRB, Kobe University Hospital IRB, and Nara Prefectural Mimuro Hospital IRB. The IRB for COMPOSE-7 only was Oita Central IRB. Both studies were conducted in compliance with local regulatory requirements, International Conference on Harmonisation Good Clinical Practice Guideline, and the Declaration of Helsinki. All patients provided written informed consent prior to participation in either study and were enrolled after the studies had been registered on www.ClinicalTrials.jp (COMPOSE-6, JAPIC CTI 142443 [February 14, 2014]; COMPOSE-7, JAPIC CTI 142607 [July 8, 2014]).

In both studies, patients received once-daily oral naldemedine at a dose of $0.2 \mathrm{mg}$ (Shionogi \& Co., Ltd., Osaka, Japan) for 48 weeks, which was divided into 2-week and 46-week treatment periods. In COMPOSE-6, patients received regular-use opioids and in COMPOSE-7, patients received $\mathrm{PR}$ oxycodone for management of chronic noncancer pain. The COMPOSE-7 study also consisted of a preliminary enrollment phase of 4-12 weeks, during which patients were switched from regular-use opioids or nonopioid analgesics to PR oxycodone prior to initiating treatment with naldemedine. Types of regular-use opioids allowed in COMPOSE-6 were any opioids approved in Japan for chronic noncancer pain, such as morphine, codeine, and fentanyl. Tramadol was allowed only if used with other regular-use opioids. In COMPOSE-6 and COMPOSE-7, dose reductions 
to naldemedine $0.1 \mathrm{mg}$ or temporary discontinuations of naldemedine were allowed during the 46-week treatment period. A regular-use laxative regimen was maintained in patients who routinely received laxatives in both studies.

Eligible patients in COMPOSE-6 (aged $\geq 20$ years) had chronic ( $\geq 3$ months) noncancer pain, had received $100 \%$ $150 \%$ of the dose of regular-use opioids for 2 weeks prior to enrollment, and had OIC. Eligible patients in COMPOSE-7 (aged $\geq 20$ years to $<80$ years) had chronic ( $\geq 3$ months) noncancer pain and OIC, and were successfully switched from regular-use opioids or nonopioid analgesics to a stable dose of PR oxycodone for 2 weeks prior to initiating treatment with naldemedine. The diagnostic threshold for OIC for both studies was five or fewer spontaneous bowel movements (SBMs) during the 2 weeks prior to enrollment and experiencing straining, a sensation of incomplete evacuation, or passage of hard pellets in $\geq 25 \%$ of all BMs. ${ }^{22}$ For both studies, patients were excluded if they had never taken laxatives for OIC, had current or previous cancer, or had a condition other than OIC that could have contributed to abnormal bowel function.

The primary objective for COMPOSE- 6 and COMPOSE-7 was to determine the long-term safety of naldemedine in patients with OIC who were receiving regular-use opioids or PR oxycodone, respectively, for chronic noncancer pain. The primary end points of COMPOSE-6 and COMPOSE-7 were summary measures of treatment-emergent adverse events (TEAEs) defined by the Medical Dictionary for Regulatory Activities (MedDRA) version 16.1. The incidence of major adverse cardiac events (MACEs), defined using standardized MedDRA queries, was also recorded in both studies. In COMPOSE-6, pain intensity was assessed using the numeric rating scale daily from baseline to week 2 and every 12 weeks from week 12 to week 48. In COMPOSE-7, pain intensity was assessed using the 24hour average Brief Pain Inventory severity score daily from baseline to week 2 and every 4 weeks from week 4 to week 48. In both studies, opioid withdrawal was assessed using Clinical Opioid Withdrawal Scale (COWS) scores at baseline and weeks 2, 12, 24, 36, and 48.

Secondary efficacy end points in both studies included the proportion of SBM responders or complete SBM (CSBM) responders during the 2-week treatment period. An SBM responder was defined as a patient with three or more SBMs per week and an increase of one or more SBMs per week from baseline (2-week period prior to enrollment). A CSBM responder was defined as a patient with an SBM that was associated with a feeling of complete bowel evacuation. Efficacy was further assessed by mean weekly change from baseline to the end of the 2-week treatment period in SBMs,
CSBMs, and SBMs without straining. In both studies, Patient Assessment of Constipation-Symptoms (PAC-SYM) and PAC-QOL scores were recorded at baseline, week 2, and every 4 weeks from week 4 to week 48 . Post hoc analyses on the proportion of SBM responders by type of opioid therapy (oxycodone, morphine, fentanyl, or other) and daily dose of opioids ( $>60 \mathrm{mg}$ or $\leq 60 \mathrm{mg}$ ) were performed on the combined populations of patients in COMPOSE-6 and COMPOSE-7.

In Japan, a limited number of patients are prescribed opioids for the treatment of noncancer pain, and at the time of these studies PR oxycodone was not approved for the treatment of patients with noncancer pain. For these reasons, enrollment was expected to be challenging. Based on these issues, the planned sample size for COMPOSE-6 was 40 enrolled patients and approximately ten patients for COMPOSE-7. In COMPOSE-6, safety analyses were performed in the safety population, defined as all patients who had received one or more doses of the study drug. The COMPOSE-7 study consisted of two separate safety populations, of which this manuscript reports only safety analyses from safety population 1, which is defined as all patients who received one or more doses of naldemedine. Efficacy was evaluated in the full-analysis set of COMPOSE-6 and COMPOSE-7. Full-analysis populations included all enrolled patients who received one or more doses of naldemedine, received an evaluation of OIC at baseline, and had one or more evaluations of OIC after the initiation of treatment.

Using the Clopper-Pearson method, 95\% CIs for TEAEs and proportions of SBM or CSBM responders were calculated. Mean changes from baseline in PAC-SYM and PACQOL scores to each assessed time point were analyzed using a paired $t$-test. Statistical significance for all analyses was set at 0.05 using two-sided tests without multiplicity adjustments. Statistical analyses were performed using SAS version 9.2 or higher (SAS Institute Inc., Cary, NC, USA).

\section{Results}

In COMPOSE-6, 43 patients were enrolled between February 24, 2014 and September 30, 2015, of which 72\% (31 of 43) completed the 48-week treatment period (Figure 1). In COMPOSE-7, ten patients were enrolled between June 30, 2014 and November 9, 2015, of which 70\% (seven of ten) completed the 48 -week treatment period (Figure 1). All patients were Asian, with a mean of fewer than two SBMs per week at baseline (Table 1). At baseline, the mean ( \pm SD) total daily morphine-equivalent dose of regularuse opioids was $74.7 \pm 68.6 \mathrm{mg}$ and PR oxycodone was $45.3 \pm 20.4 \mathrm{mg}$ (Table 1$)$. 

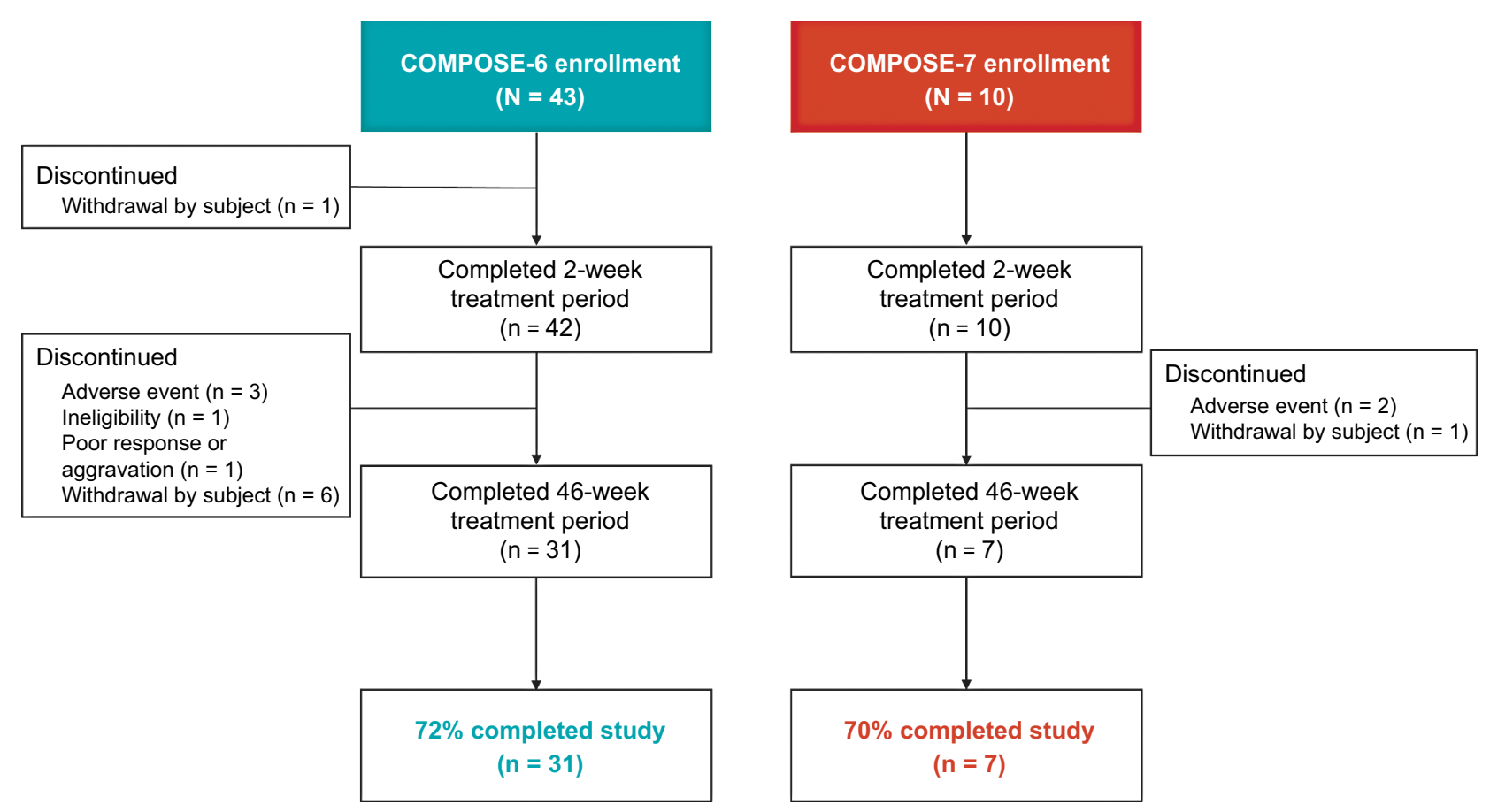

Figure I Patient disposition for the COMPOSE-6 and COMPOSE-7 studies.

Table I Patient demographics and baseline characteristics in COMPOSE-6 and COMPOSE-7 (FAS, except where noted)

\begin{tabular}{|c|c|c|}
\hline Parameter & COMPOSE-6 $(n=42)$ & COMPOSE-7 $(n=10)$ \\
\hline Mean age, years (SD) & $63.9(14.6)$ & $66.9(7.4)$ \\
\hline \multicolumn{3}{|l|}{ Sex, n (\%) } \\
\hline Female & $23(55)$ & $8(80)$ \\
\hline Male & $19(45)$ & $2(20)$ \\
\hline \multicolumn{3}{|l|}{ Race, n (\%) } \\
\hline Asian & $42(100)$ & $10(100)$ \\
\hline BMI, kg/m² (SD) & $22.3(3.8)$ & $22.7(3.2)$ \\
\hline Mean SBMs per week (SD) & $1.21(0.90)$ & $1.30(0.82)$ \\
\hline \multicolumn{3}{|c|}{ Mean total daily dose, $\mathrm{mg}(\mathrm{SD})^{\mathrm{a}}$} \\
\hline Regular-use opioids & $74.7(68.6)$ & - \\
\hline PR oxycodone & - & $45.3(20.4)$ \\
\hline \multicolumn{3}{|l|}{ Prior use, n (\%) } \\
\hline Routine laxatives & $37(86)$ & $9(90)$ \\
\hline Rescue laxatives & $39(91)$ & $\mathrm{I}(\mathrm{I0})$ \\
\hline \multicolumn{3}{|c|}{ Concomitant regular-use laxatives, $\mathbf{n}(\%)^{\mathrm{b}}$} \\
\hline Magnesium oxide & $32(74)$ & $8(80)$ \\
\hline Sennoside A + B & II (26) & $\mathrm{I}(10)$ \\
\hline Sodium picosulfate & $2(5)$ & - \\
\hline Daikenchuto & $2(5)$ & - \\
\hline Tokakujokito & $2(5)$ & $\mathrm{I}(\mathrm{I0})$ \\
\hline Senna alexandrina & I (2) & - \\
\hline Alosenn & I (2) & - \\
\hline Circanetten & I (2) & - \\
\hline Rheum palmatum & I (2) & - \\
\hline Lubiprostone & $I(2)$ & $\mathrm{I}(\mathrm{I0})$ \\
\hline Daiokanzoto & I (2) & - \\
\hline Mashiningan & I (2) & - \\
\hline Junchoto & I (2) & - \\
\hline
\end{tabular}

Notes: "Oral morphine equivalents; 'safety populations ( $\mathrm{N}=43$ for COMPOSE-6 and $\mathrm{N}=10$ for COMPOSE-7 [safety population I]). "-" indicates that a value was not applicable for one study or the other. For example, in COMPOSE-6, patients received regular-use opioids and in COMPOSE-7, patients received PR oxycodone. Additionally, several 'regular use laxatives' used in COMPOSE-6 were not used in COMPOSE-7.

Abbreviations: BMI, body-mass index; FAS, full-analysis set; PR, prolonged release; SBM, spontaneous bowel movement. 
Side effects that occurred with once-daily naldemedine for 48 weeks were mostly mild or moderate in severity in patients with OIC who were receiving regular-use opioid therapy or PR oxycodone for chronic noncancer pain (Table 2). Although TEAEs were reported in $88 \%$ (38 of 43 ) and $90 \%$ (nine of ten) of patients in COMPOSE- 6 and COMPOSE-7, respectively, treatment-related AEs occurred in only $28 \%$ of patients in COMPOSE- 6 and $50 \%$ of patients in COMPOSE-7 (Table 2). Importantly, none of the significant AEs occurring in either COMPOSE-6 (one each for anal fissure, diarrhea, and decreased blood potassium) or COMPOSE-7 (one each for malaise, increased blood triglycerides, and protein urine present) were considered related to the study drug. None of the serious AEs in COMPOSE-6 (one

Table 2 Summary of safety in COMPOSE-6 (safety population) and COMPOSE-7 (safety population I)

\begin{tabular}{|c|c|c|}
\hline Safety parameter, n (\%) & COMPOSE-6 $(\mathrm{N}=43)$ & COMPOSE-7 $(\mathbf{N}=10)$ \\
\hline TEAEs & $38(88)$ & $9(90)$ \\
\hline $95 \% \mathrm{Cl}$ & $74.9-96.1$ & $55.5-99.7$ \\
\hline Treatment-related AEs & $12(28)$ & $5(50)$ \\
\hline $95 \% \mathrm{Cl}$ & $15.3-43.7$ & $|8.7-8| .3$ \\
\hline Significant AEs ${ }^{\mathrm{a}}$ & $3(7)$ & $2(20)$ \\
\hline $95 \% \mathrm{Cl}$ & $1.5-19.1$ & $2.5-55.6$ \\
\hline AEs leading to study withdrawal & $3(7)$ & $\mathrm{I}(10)^{\mathrm{b}}$ \\
\hline $95 \% \mathrm{Cl}$ & $1.5-19.1$ & $0.3-44.5$ \\
\hline Serious AEs (except death) ${ }^{c}$ & $4(9)$ & 0 \\
\hline $95 \% \mathrm{Cl}$ & $2.6-22.1$ & - \\
\hline Deaths & $\mathrm{I}(2)^{\mathrm{d}}$ & 0 \\
\hline $95 \% \mathrm{Cl}$ & $0.1-12.3$ & - \\
\hline \multicolumn{3}{|l|}{ TEAEs by SOC and PT that occurred in $\geq 2$ patients } \\
\hline Gastrointestinal disorders & $23(53)$ & $5(50)$ \\
\hline Diarrhea & $10(23)$ & $4(40)$ \\
\hline Nausea & $5(12)$ & $\mathrm{I}(10)$ \\
\hline Abdominal pain & $4(9)$ & $\mathrm{I}(\mathrm{I0})$ \\
\hline Vomiting & $4(9)$ & $\mathrm{I}(\mathrm{I0})$ \\
\hline Infections and infestations & $18(42)$ & $3(30)$ \\
\hline Nasopharyngitis & II (26) & $3(30)$ \\
\hline Gastroenteritis & $2(5)$ & 0 \\
\hline Influenza & $2(5)$ & 0 \\
\hline Nervous system disorders & $8(19)$ & $\mathrm{I}(\mathrm{I0})$ \\
\hline Somnolence & $3(7)$ & $\mathrm{I}(\mathrm{I0})$ \\
\hline Dizziness & $2(5)$ & 0 \\
\hline General disorders and administration-site conditions & $5(12)$ & $2(20)$ \\
\hline Malaise & $\mathrm{I}(2)$ & $2(20)$ \\
\hline Investigations & $5(12)$ & $2(20)$ \\
\hline Blood creatine phosphokinase increase & $2(5)$ & $2(20)$ \\
\hline Injury, poisoning, and procedural complications & $6(14)$ & 0 \\
\hline Contusion & $3(7)$ & 0 \\
\hline Fall & $2(5)$ & 0 \\
\hline Ligament sprain & $2(5)$ & 0 \\
\hline Metabolism and nutrition disorders & $4(9)$ & 0 \\
\hline Decreased appetite & $3(7)$ & 0 \\
\hline Skin and subcutaneous tissue disorders & $4(9)$ & 0 \\
\hline Eczema & $2(5)$ & 0 \\
\hline Psychiatric disorders & $2(5)$ & $2(20)$ \\
\hline Anxiety & $2(5)$ & $2(20)$ \\
\hline
\end{tabular}

Notes: aSignificant AEs were defined as any AE that led to withdrawal or was severe, other than a serious AE. Severe AEs were CTCAE grade 3 or higher, caused interruption of the patient's usual daily activities, or had a clinically significant effect. 'In COMPOSE-7, two patients withdrew from the study. However, given that one TEAE occurred during the preliminary screening phase, only one patient was considered to have a TEAE leading to study withdrawal. 'Serious AEs were defined as any AE occurring at any dose that resulted in death, life-threatening condition, hospitalization or prolongation of existing hospitalization, persistent or significant disability or incapacity, congenital anomaly or birth defect, or other medically important conditions. Important medical events that might not result in death, were not life-threatening, or did not require hospitalization were considered serious AEs when (based upon appropriate medical judgment) they jeopardized the patient and required medical intervention to prevent one of the aforementioned outcomes. 'Suspected cause of death was related to venous thrombosis in the lower extremities and pulmonary embolism, which was present prior to screening. "-" Indicates that there is no $95 \%$ Cl calculated for 'Serious AEs' or 'Deaths', as neither occurred in the COMPOSE-7 study.

Abbreviations: $A E$, adverse event; CTCAE, Common Terminology Criteria for Adverse Events; PT, preferred term; SOC, system-organ class; TEAE, treatment-emergent adverse events. 
each for urethral stenosis, cerebral infarction, cholelithiasis, ileus, and urinary tract infection) were considered related to naldemedine, and there were no serious AEs reported in COMPOSE-7. In both studies, diarrhea and nasopharyngitis were the most frequently reported TEAEs (Table 2). The TEAE of diarrhea was observed mainly at the initiation of naldemedine treatment, and the majority of cases were considered mild or moderate in severity in both studies. No trends in the timing of onset of the TEAE nasopharyngitis were observed. All cases of nasopharyngitis were mild or moderate in severity and considered unrelated to the study drug.

Diarrhea was the most frequently reported treatmentrelated AE in COMPOSE-6 (14\% [six of 43 patients]) and COMPOSE-7 (40\% [four of ten patients]). In COMPOSE-6, TEAEs of anal fissure, ileus, and death led to study discontinuation of three patients, respectively. The death was suspected to be associated with venous thrombosis in the lower extremities and pulmonary embolism, which were present prior to enrollment. In COMPOSE-7, one patient withdrew from the study due to a TEAE of malaise. None of the TEAEs leading to study withdrawal in either study were considered related to naldemedine. In COMPOSE-6, MACEs occurred in 7\% (three of 43 ) of patients. The occurrence of increased blood creatine phosphokinase levels $(n=2)$ and cerebral infarction $(n=1)$ was considered unrelated to the study drug. No MACEs occurred in COMPOSE-7.

No significant clinical changes in pain intensity from baseline were observed over the treatment periods in either study (Figure 2). Moreover, daily mean ( \pm SD) morphine-equivalent doses of opioids changed minimally from baseline during the overall treatment period in COMPOSE-6 $(1.85 \pm 10.71 \mathrm{mg})$ and COMPOSE-7 (6.52 $\pm 16.53 \mathrm{mg})$. Mean total COWS scores were moderately stable from baseline to all assessed time points in COMPOSE-6 and COMPOSE-7 (Figure 3).

Proportions of SBM responders were $81 \%$ (34 of 42) in COMPOSE-6 and 90\% (nine of ten) in COMPOSE-7
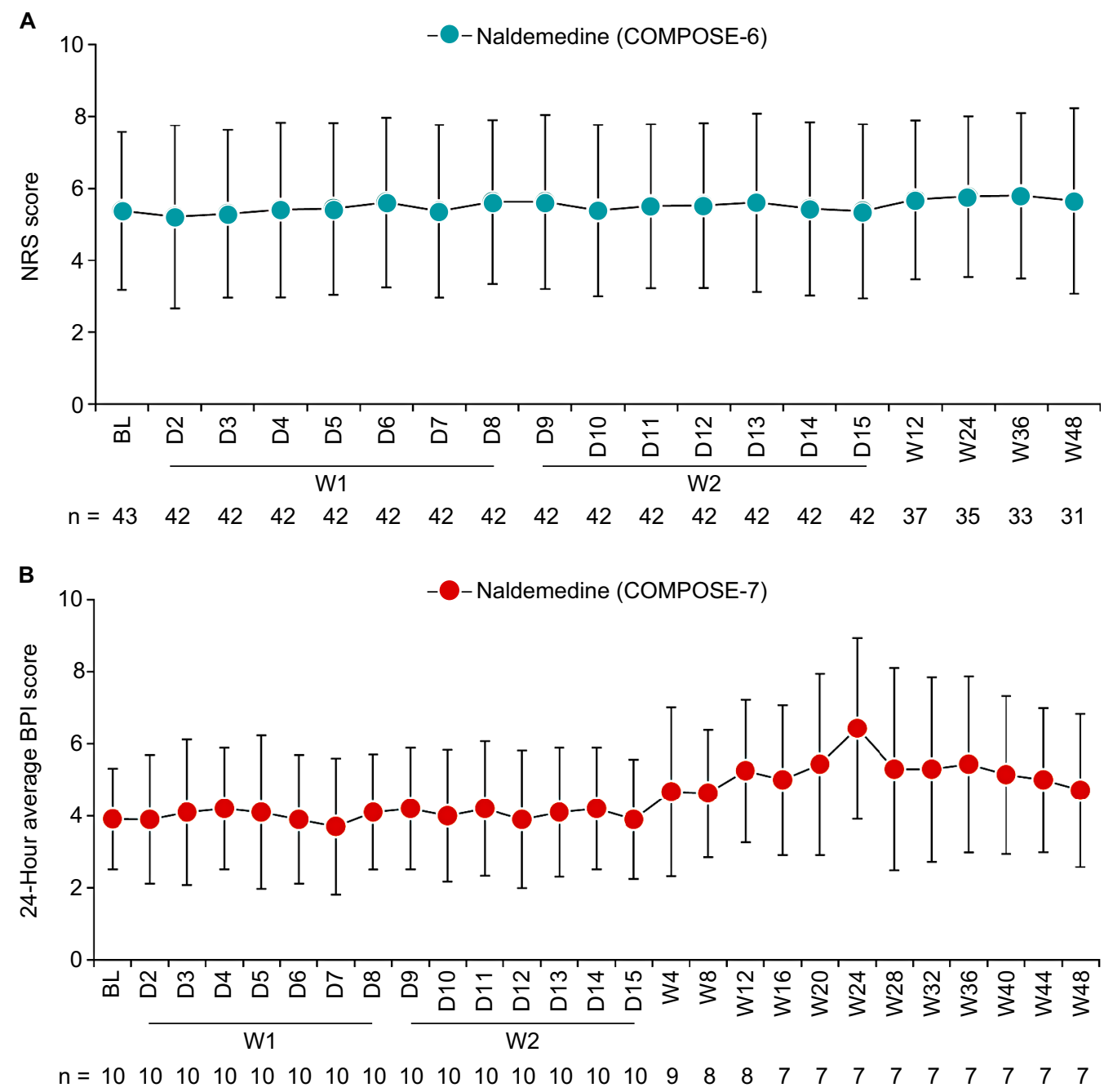

Figure 2 (A) NRS scores in COMPOSE-6 (safety population) and (B) 24-hour average BPI scores in COMPOSE-7 (safety population I).

Notes: All data are mean \pm SD.

Abbreviations: BL, baseline; BPI, Brief Pain Inventory; D, day; NRS, numeric rating scale; W, week. 

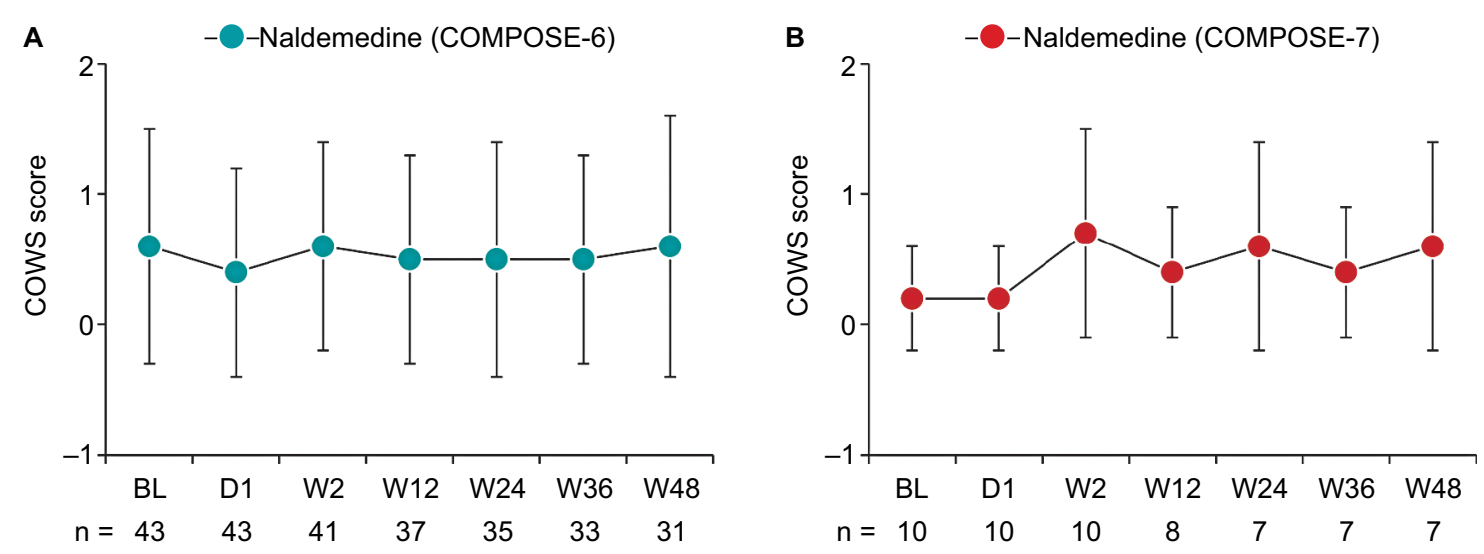

Figure 3 COWS scores in (A) COMPOSE-6 (safety population) and (B) COMPOSE-7 (safety population I) studies.

Notes: All data are mean \pm SD.

Abbreviations: BL, baseline; COWS, Clinical Opioid Withdrawal Scale; D, day; W, week.
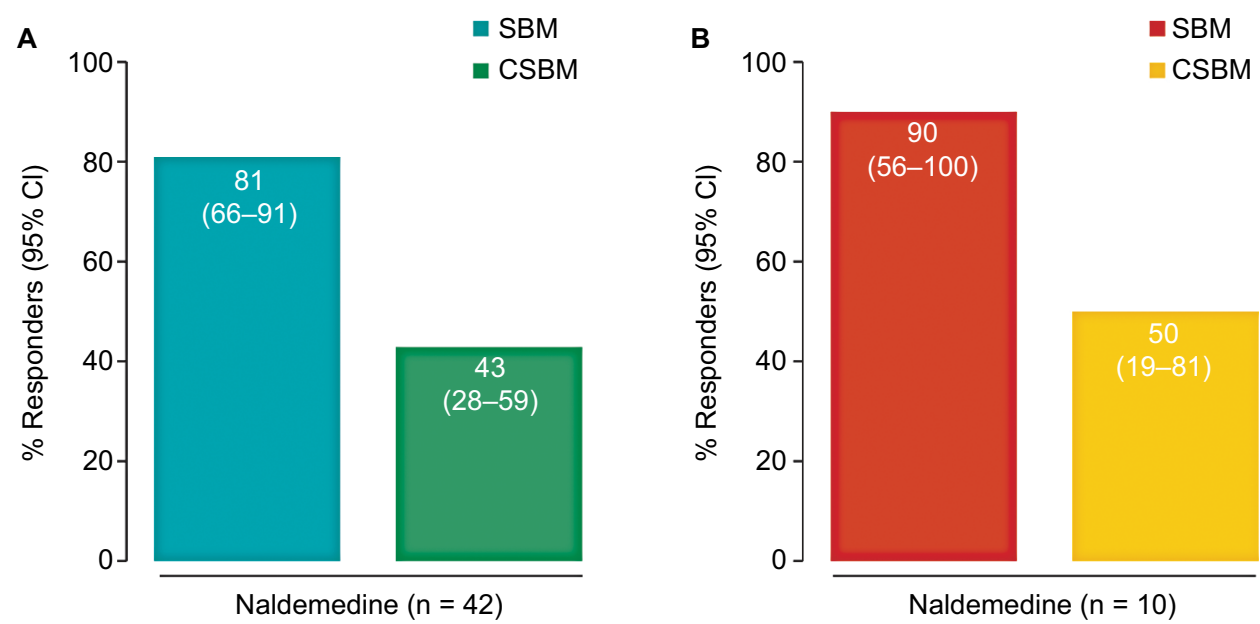

Figure 4 Proportions of SBM and CSBM responders at the end of the 2-week treatment period in (A) COMPOSE-6 and (B) COMPOSE-7 (both FAS). Notes: All data are percents $(95 \% \mathrm{Cl})$.

Abbreviations: CSBM, complete spontaneous bowel movement; FAS, full-analysis set; SBM, spontaneous bowel movement.

(Figure 4A). Proportions of CSBM responders were $43 \%$ (18 of 42) in COMPOSE- 6 and 50\% (five of ten) in COMPOSE-7 (Figure 4B). A mean increase from baseline to the end of the 2-week treatment period in weekly frequency of SBMs, CSBMs, and SBMs without straining was observed in both studies (Figure 5). The proportion of SBM responders in the combined population of patients in COMPOSE- 6 and COMPOSE-7 $(\mathrm{N}=52$ ) was $83 \%$ (43 of 52). Subgroup analysis by opioid type revealed similar and high proportions $(\geq 73 \%)$ of SBM responders, regardless of the type of opioid received (Figure S1A) or the daily dose of opioids (Figure S1B).

In COMPOSE-6, a significant decrease (improvement) from baseline was observed in overall PAC-SYM scores and scores for each domain at week $2(P \leq 0.0052)$ and week 48 ( $P \leq 0.0005$; Figure 6A). Similarly, in COMPOSE-7, significant decreases from baseline in overall PAC-SYM scores and scores for each domain were observed at week $2(P \leq 0.0250)$ and week $48(P \leq 0.0389$; Figure 6B). In COMPOSE-6, a significant decrease from baseline was observed in overall PAC-QOL scores and scores for each domain at week $2(P<0.0001)$ and week $48(P<0.0001$; Figure 7A). In COMPOSE-7, significant decreases from baseline in overall PAC-QOL scores and scores for all domains were observed at week $2(P \leq 0.0063)$ and for all domains at week 48 ( $P \leq 0.0470$; Figure 7B), except for the domain of physical discomfort. 

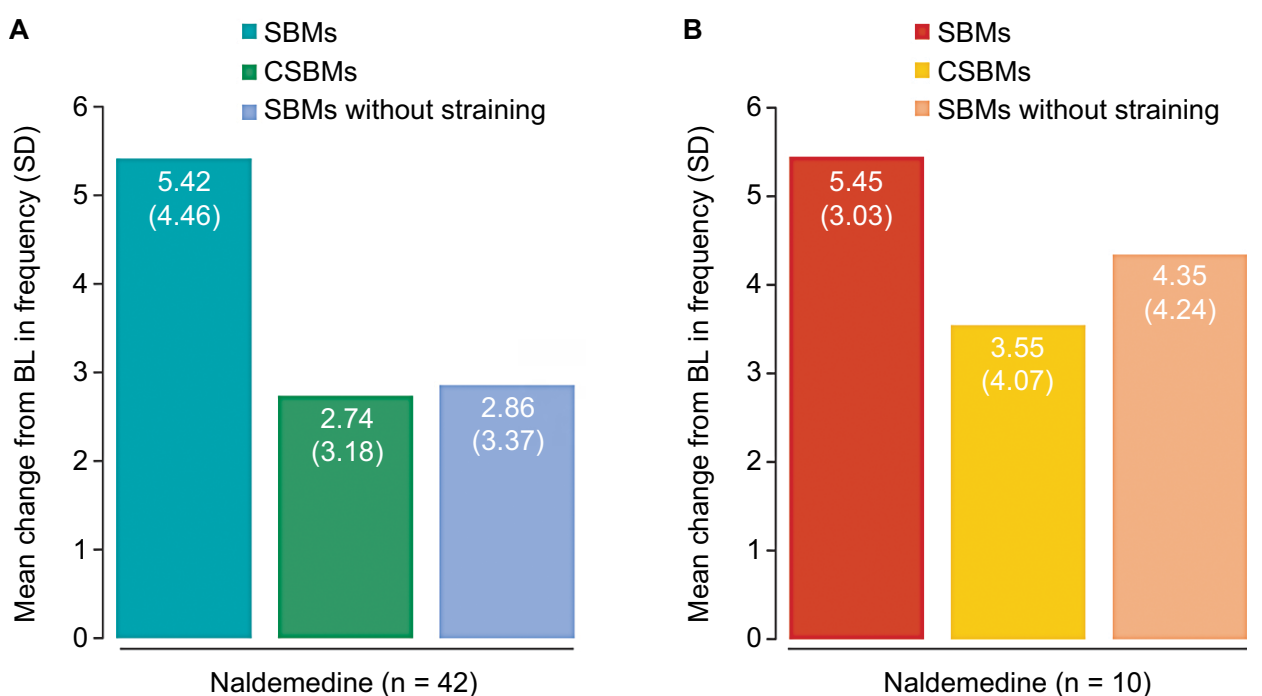

Figure 5 Change from baseline in frequency of SBMs, CSBMs, and SBMs without straining per week at the end of the 2-week treatment period in (A) COMPOSE-6 and (B) COMPOSE-7 (both FAS).

Notes: All data are mean (SD)

Abbreviations: BL, baseline; CSBMs, complete spontaneous bowel movements; FAS, full-analysis set; SBMs, spontaneous bowel movements.

A

Naldemedine (COMPOSE-6)

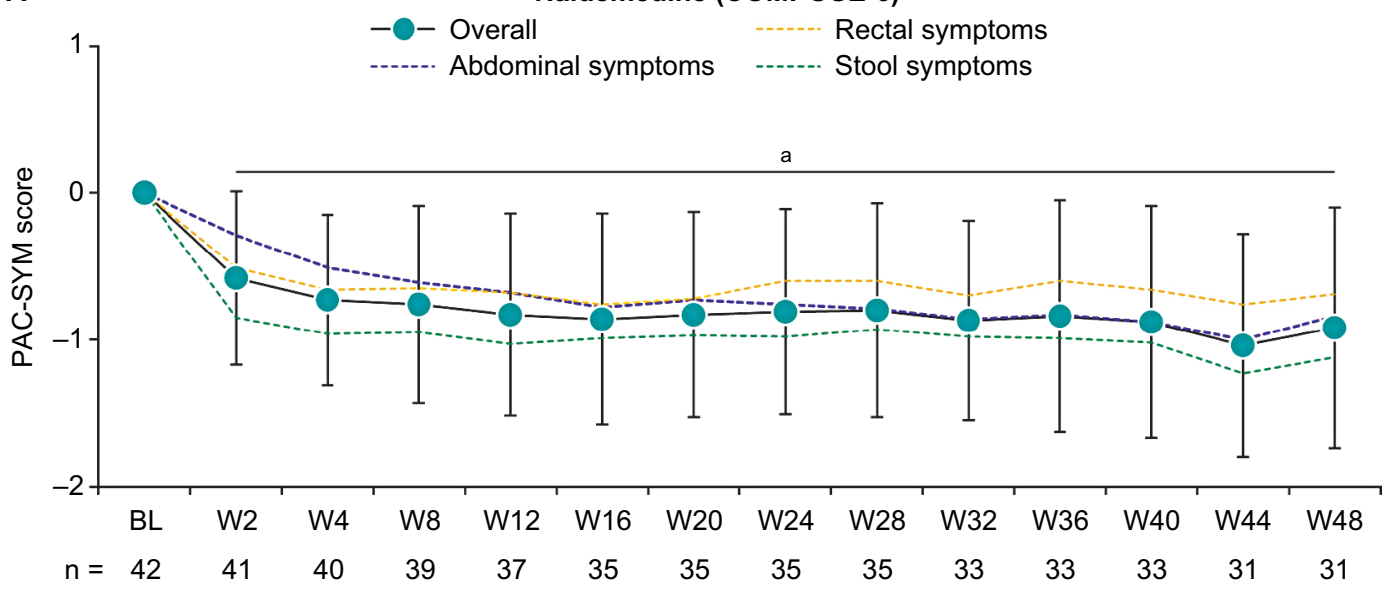

B

Naldemedine (COMPOSE-7)

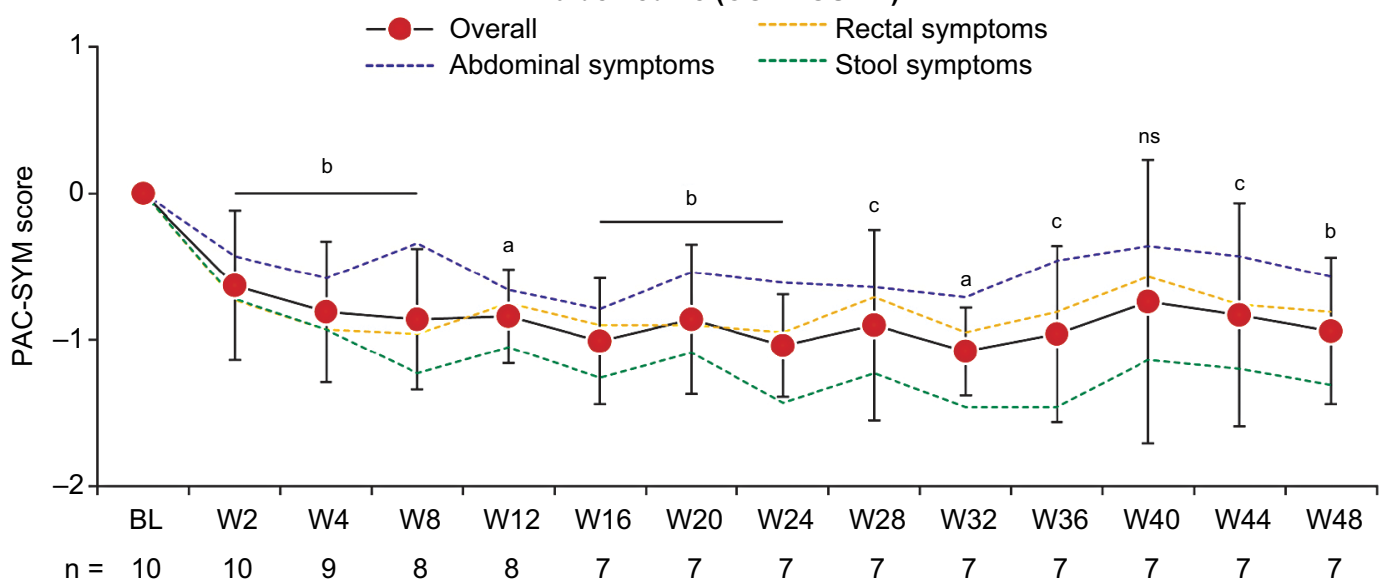

Figure 6 Change from baseline in PAC-SYM overall scores and scores for each domain over the treatment periods in (A) COMPOSE-6 and (B) COMPOSE-7 (both FAS) Notes: ${ }^{a} P<0.0001$; ${ }^{b} P<0.005$; ${ }^{C P}<0.05$ vs $B L$ for overall scores. All data are mean $\pm S D$ for overall scores.

Abbreviations: BL, baseline; FAS, full-analysis set; ns, not significant; PAC-SYM, Patient Assessment of Constipation-Symptoms; W, week. 

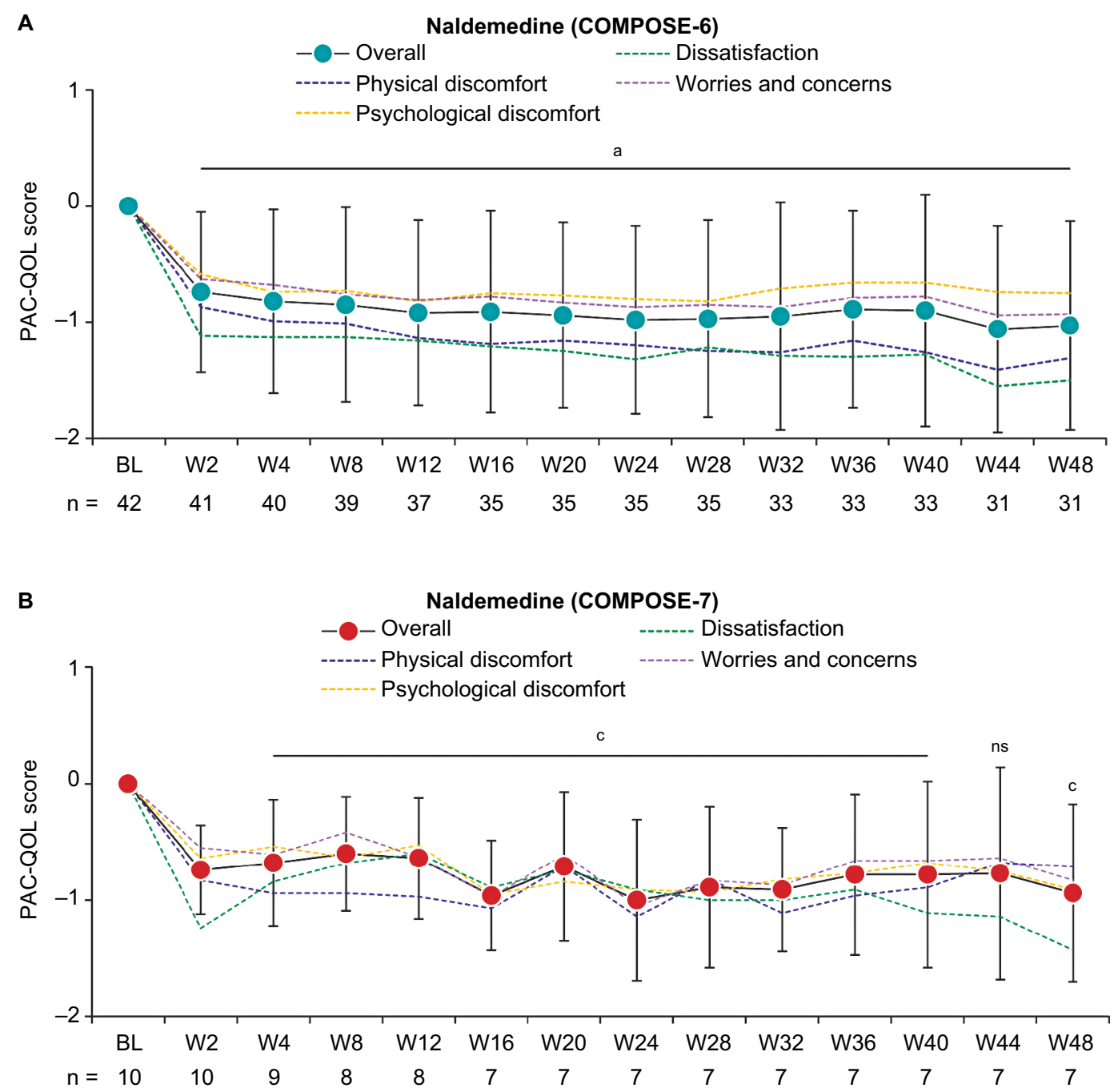

Figure 7 Mean change from baseline in PAC-QOL overall scores and scores for each domain in (A) COMPOSE-6 and (B) COMPOSE-7 (both FAS). Notes: ${ }^{a} P<0.0001 ; \quad P P<0.005 ; c P<0.05$ vs $B L$ for overall scores. All data are mean $\pm S D$ for overall scores.

Abbreviations: BL, baseline; FAS, full-analysis set; ns, not significant; PAC-QOL, Patient Assessment of Constipation-Quality of Life; W, week.

\section{Discussion}

Side effects that occurred with once-daily oral naldemedine $0.2 \mathrm{mg}$ for 48 weeks were mostly mild or moderate in severity in Japanese patients with OIC who were receiving regular-use opioids or PR oxycodone for chronic noncancer pain. Naldemedine did not impede opioid analgesia and was not associated with signs or symptoms of opioid withdrawal throughout the studies. Improvements in bowel function were observed within 2 weeks of treatment with naldemedine in these study populations. Moreover, treatment with naldemedine was associated with significant, long-term improvements in patientreported outcomes on constipation-related symptoms and QOL.

In both studies, diarrhea and nasopharyngitis were the most frequently reported TEAEs. The TEAE of diarrhea was mainly observed during the first 2 weeks of treatment, and almost all cases were mild or moderate in severity, suggesting that diarrhea did not have a major impact on the tolerability of naldemedine. Nasopharyngitis, which could be a manifestation of opioid withdrawal, was unlikely to be a major safety concern for naldemedine because all cases were considered unrelated to naldemedine. Moreover, the incidence of nasopharyngitis was not associated with a trend regarding the initiation of naldemedine treatment. The mechanism of action of PAMORAs ${ }^{17}$ and observations from previous naldemedine studies conducted in patients with chronic noncancer pain, ${ }^{18}$ as well as in Japanese patients with cancer pain, ${ }^{19}$ are consistent with the incidence of gastrointestinal disorders observed in the COMPOSE-6 and COMPOSE-7 studies. Furthermore, clinical studies with other PAMORAs, such as naloxegol and methylnaltrexone bromide, have reported an increased incidence of gastrointestinal disorders. ${ }^{23-25}$ 
Favorable tolerability of naldemedine was further demonstrated by patient disposition in both COMPOSE-6 and COMPOSE-7. Overall, over 70\% of patients completed both studies. In COMPOSE-6, patients withdrew mainly during the 46-week treatment period $(n=11)$ and only one patient during the 2-week treatment period. In COMPOSE-7, patients discontinued treatment only during the 46-week treatment period. Furthermore, only five patients in total withdrew because of AEs in either COMPOSE-6 or COMPOSE-7, whereas ten patients discontinued treatment because of ineligibility, poor response or aggravation, or withdrawal by subject.

An important outcome of these studies was that the assessments of pain intensity and opioid withdrawal showed little change throughout the 48 -week treatment periods. Moreover, the dose of PR oxycodone required to manage pain levels was stable for the duration of COMPOSE-7. These results suggest that coadministration of naldemedine and PR oxycodone for the treatment of OIC in patients with chronic noncancer pain does not introduce novel safety concerns or hinder the analgesic benefits of the opioid therapy. Although postmarketing surveys in Japan can help confirm results of COMPOSE-6, results related to PR oxycodone are difficult to confirm because this therapy is not currently approved for patients with noncancer pain in Japan.

Administration of naldemedine for 2 weeks effectively treated OIC, as evidenced by the high proportions of SBM and CSBM responders and increases from baseline in the weekly frequency of SBMs, CSBMs, and SBMs without straining. The 2-week efficacy results in these studies are similar to those observed from previous randomized, doubleblind, placebo-controlled, Phase III studies evaluating the efficacy of naldemedine in patients with OIC and cancer pain $^{19}$ or OIC and chronic noncancer pain. ${ }^{18}$ Subgroup analyses by type of opioid and dose of opioids on the pooled population of patients in COMPOSE-6 and COMPOSE-7 showed similar and high proportions of SBM responders, irrespective of the type of opioid used or the daily dose. These results suggest that naldemedine can improve bowel function in patients with OIC without requiring adjustments to the existing opioid regimen used for the management of chronic noncancer pain.

Limitations to these studies included relatively small patient populations, the lack of a placebo control, and the absence of a diverse patient population. The safety and efficacy of naldemedine have previously been demonstrated in two large, non-Japanese, randomized, double-blind, placebo-controlled, 12-week Phase III clinical studies. ${ }^{18}$
As such, we believe that the results from COMPOSE-6 and COMPOSE-7 are important additions to the body of knowledge on the long-term use of naldemedine in Japanese patients.

\section{Conclusion}

In these open-label Phase III clinical studies, side effects occurring with once-daily oral naldemedine $0.2 \mathrm{mg}$ for 48 weeks in Japanese patients with OIC who were receiving regular-use opioids or PR oxycodone for chronic noncancer pain were mostly mild or moderate in severity. Moreover, concomitant treatment with naldemedine did not interfere with the analgesic effects of opioids or precipitate any signs or symptoms of opioid withdrawal. The results suggest that treatment with naldemedine can improve bowel function and constipation-related QOL in this study population. Together, the safety and efficacy results from the COMPOSE-6 and COMPOSE-7 studies support the long-term use of naldemedine for OIC in Japanese patients receiving regularuse opioids or PR oxycodone for chronic noncancer pain.

\section{Acknowledgments}

This study was funded by Shionogi. Interim data from this work were presented in part at the 51st Annual Meeting of the Japan Society of Pain Clinicians in Gifu, Japan, July 21, 2017. V Ruvini Jayasinghe, PhD of Oxford PharmaGenesis Inc, Newtown, PA, USA provided editorial assistance, which was funded by Shionogi \& Co., Ltd, Osaka, Japan.

\section{Author contributions}

All authors contributed to data analysis, drafting and revising the article, gave final approval of the version to be published, and agree to be accountable for all aspects of the work. YS and MS contributed to data collection. YS, TY, MA, and YT contributed to the study design. YT contributed to statistical data analysis.

\section{Disclosure}

TY, MA, and YT are employees of Shionogi. MS has served as an advisor for Shionogi and Janssen Pharmaceutical, a consultant for Hisamitsu Pharmaceutical and Toray Medical, and has received honoraria from Shionogi, Daiichi Sankyo, Eli Lilly Japan, Nihon Zoki, and Pfizer Japan. YS has received research funding and personal fees from Shionogi, Asahi Kasei, Daiichi Sankyo, Kyowa Kirin, Pfizer, Nippon Zoki Pharmaceutical, and Tsumura, and has received personal fees from Janssen Pharmaceutical, Hisamitsu Pharmaceutical, and Ayumi Pharmaceutical. The authors report no other conflicts of interest in this work. 


\section{References}

1. Onishi E, Kobayashi T, Dexter E, Marino M, Maeno T, Deyo RA. Comparison of opioid prescribing patterns in the United States and Japan: primary care physicians' attitudes and perceptions. J Am Board Fam Med. 2017;30(2):248-254.

2. Inoue S, Kobayashi F, Nishihara M, et al. Chronic pain in the Japanese community - prevalence, characteristics and impact on quality of life. PLoS One. 2015;10(6):e0129262.

3. Morlion BJ, Mueller-Lissner SA, Vellucci R, et al. Oral prolongedrelease oxycodone/naloxone for managing pain and opioid-induced constipation: a review of the evidence. Pain Pract. 2018;18(5):647-665.

4. Huang L, Zhou JG, Zhang Y, et al. Opioid-induced constipation relief from fixed-ratio combination prolonged-release oxycodone/naloxone compared with oxycodone and morphine for chronic nonmalignant pain: a systematic review and meta-analysis of randomized controlled trials. J Pain Symptom Manage. 2017;54(5):737-748.e3.

5. Kalso E, Edwards JE, Moore RA, McQuay HJ. Opioids in chronic non-cancer pain: systematic review of efficacy and safety. Pain. 2004;112(3):372-380.

6. Coyne KS, Margolis MK, Yeomans K, et al. Opioid-induced constipation among patients with chronic noncancer pain in the United States, Canada, Germany, and the United Kingdom: laxative use, response, and symptom burden over time. Pain Med. 2015;16(8):1551-1565.

7. Bell TJ, Panchal SJ, Miaskowski C, Bolge SC, Milanova T, Williamson R. The prevalence, severity, and impact of opioid-induced bowel dysfunction: results of a US and European Patient Survey (PROBE 1). Pain Med. 2009;10(1):35-42.

8. Gupta S, Patel H, Scopel J, Mody RR. Impact of constipation on opioid therapy management among long-term opioid users, based on a patient survey. J Opioid Manag. 2015;11(4):325-338.

9. Pergolizzi JV Jr, Raffa RB, Pappagallo M, et al. Peripherally acting $\mu$-opioid receptor antagonists as treatment options for constipation in noncancer pain patients on chronic opioid therapy. Patient Prefer Adherence. 2017;11:107-119.

10. Coyne KS, Sexton C, Locasale RJ, King FR, Margolis MK, Ahmedzai $\mathrm{SH}$. Opioid-induced constipation among a convenience sample of patients with cancer pain. Front Oncol. 2016;6:131.

11. Emmanuel A, Johnson M, McSkimming P, Dickerson S. Laxatives do not improve symptoms of opioid-induced constipation: results of a patient survey. Pain Med. 2017;18(10):1932-1940.

12. Holzer P. New approaches to the treatment of opioid-induced constipation. Eur Rev Med Pharmacol Sci. 2008;12 (Suppl 1):119-127.
13. Ward A, Del Campo M, Hauser K. Complications with oxycodone and naloxone. Aust Prescr. 2017;40(4):156-157.

14. Burns E, McWilliams K, Ross C. A cautionary tale of oral naloxone. J Pain Symptom Manage. 2014;47(2):e1-e2.

15. Kang JH, Lee GW, Shin SH, Bruera E. Opioid withdrawal syndrome after treatment with low-dose extended-release oxycodone and naloxone in a gastric cancer patient with portal vein thrombosis. J Pain Symptom Manage. 2013;46(2):e15-e17.

16. Symproic ${ }^{\circledR}$ (naldemedine) [package insert]. Florham Park, NJ: Shionogi Inc.; 2017.

17. Streicher JM, Bilsky EJ. Peripherally acting $\mu$-opioid receptor antagonists for the treatment of opioid-related side effects: mechanism of action and clinical implications. J Pharm Pract. 2017:10.1177/0897190017732263.

18. Hale M, Wild J, Reddy J, Yamada T, Arjona Ferreira JC. Naldemedine versus placebo for opioid-induced constipation (COMPOSE-1 and COMPOSE-2): two multicentre, phase 3, double-blind, randomised, parallel-group trials. Lancet Gastroenterol Hepatol. 2017;2(8):555-564.

19. Katakami N, Harada T, Murata T, et al. Randomized phase III and extension studies of naldemedine in patients with opioid-induced constipation and cancer. J Clin Oncol. 2017;35(34):3859-3866.

20. Saeki S. Indication and usage of opioids except morphine for chronic non-malignant intractable pain. Masui. 2008;57(11):1351-1358. [Japanese].

21. Yamaguchi T, Shima Y, Morita T, Hosoya M, Matoba M; Japanese Society of Palliative Medicine. Clinical guideline for pharmacological management of cancer pain: the Japanese Society of Palliative Medicine recommendations. Jpn J Clin Oncol. 2013;43(9):896-909.

22. Camilleri M, Drossman DA, Becker G, Webster LR, Davies AN, Mawe GM. Emerging treatments in neurogastroenterology: a multidisciplinary working group consensus statement on opioid-induced constipation. Neurogastroenterol Motil. 2014;26(10):1386-1395.

23. Webster L, Chey WD, Tack J, Lappalainen J, Diva U, Sostek M. Randomised clinical trial: the long-term safety and tolerability of naloxegol in patients with pain and opioid-induced constipation. Aliment Pharmacol Ther. 2014;40(7):771-779.

24. Chey WD, Webster L, Sostek M, Lappalainen J, Barker PN, Tack J. Naloxegol for opioid-induced constipation in patients with noncancer pain. N Engl J Med. 2014;370(25):2387-2396.

25. Rauck R, Slatkin NE, Stambler N, Harper JR, Israel RJ. Randomized, double-blind trial of oral methylnaltrexone for the treatment of opioidinduced constipation in patients with chronic noncancer pain. Pain Pract. 2017;17(6):820-828. 


\section{Supplementary material}

A

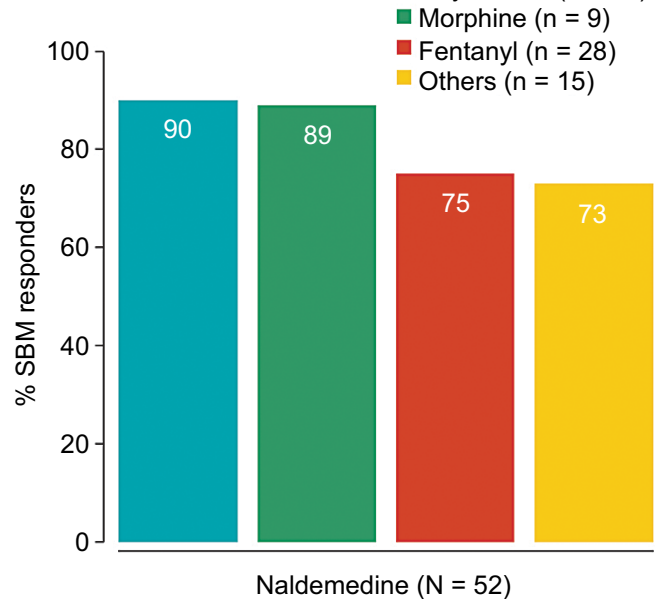

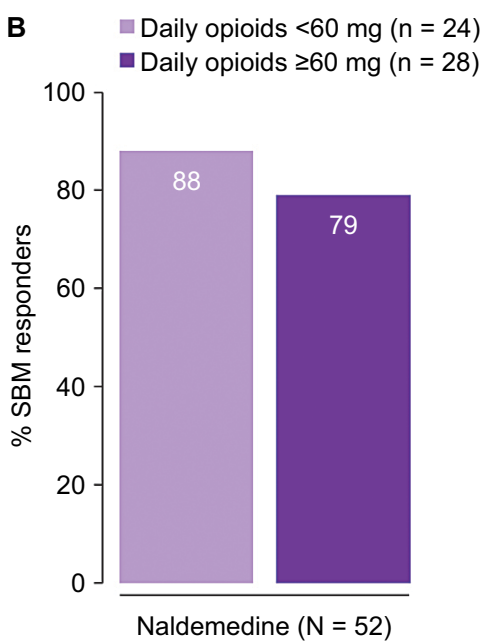

Figure SI Proportion of SBM responders in the combined population of patients in COMPOSE-6 and COMPOSE-7 by (A) type of opioid and (B) daily dose of opioid morphine equivalent at baseline.

Abbreviation: SBM, spontaneous bowel movement.

\section{Publish your work in this journal}

The Journal of Pain Research is an international, peer reviewed, open access, online journal that welcomes laboratory and clinical findings in the fields of pain research and the prevention and management of pain. Original research, reviews, symposium reports, hypothesis formation and commentaries are all considered for publication.

\section{Dovepress}

The manuscript management system is completely online and includes a very quick and fair peer-review system, which is all easy to use. Visit http://www.dovepress.com/testimonials.php to read real quotes from published authors. 